\title{
EDITORIAL
}

\section{Fabry disease}

\section{S F Nagueh}

Heart 2003;89:819-820

It is incumbent upon physicians evaluating men with unexplained left ventricular hypertrophy-particularly those without severe outflow tract obstruction - to consider Fabry disease in the differential diagnosis

$\mathrm{F}$ abry disease is a rare $\mathrm{X}$ linked recessive disorder resulting from a deficiency of the lysosomal enzyme $\alpha$ galactosidase A. Accordingly, hemizygous males have the most severe form of the disease and heterozygous females usually have a more benign presentation. ${ }^{1}$ The enzymatic defect in this lysosomal storage disease leads to the accumulation of globotriaosylceramide in several organs including the skin, kidney, nervous system, cornea, and the heart. Patients with the classic form suffer from acroparesthesias, hypohidrosis, angiokeratomas, corneal opacities, cerebrovascular lesions, cardiac disorders, and renal dysfunction. More than 160 mutations in the $\alpha$ galactosidase A gene have been reported. Depending on the mutation, the enzyme activity may be reduced or abolished. In the more benign mutations, the enzyme activity and stability are reduced but the active site is still capable of binding to the substrate. These mutant forms of the protein may be stabilised by chemical chaperones such as galactose that bind to the active site of the enzyme. ${ }^{2}$ This in turn promotes some folding and stability of the protein until it is transported into the lysosomes. There, the accumulated globotriaosylceramide molecules displace the chaperone and are hydrolysed by the enzyme. Therefore in selected patients where the enzyme active site is still capable of binding to the substrate, galactose can be used to increase the enzyme activity and decrease the accumulation of the globotriaosylceramide molecules. ${ }^{3}$

\section{CARDIAC MANIFESTATIONS OF FABRY DISEASE}

There are two forms of cardiac disease: one where the heart is affected along with other organs; and an atypical variant of the disease, with manifestations limited to the heart. In these cases, the diagnosis may be ascertained by endomyocardial biopsy and/or measurement of plasma $\alpha$ galactosidase A activity. Sarcoplasmic vacuolisation is the classic finding on light microscopy of hematoxylin and eosin stained tissue. On electron microscopy, concentric lamellar bodies are noted in the sarcoplasm of myocardial cells.

Electrocardiographic findings include a short PR interval, conduction disorders of the atrioventricular node, His bundle or its branches, ${ }^{45}$ in addition to chamber enlargement with left ventricular (LV) hypertrophy being the most frequent of these. Echocardiographic abnormalities include the presence of LV/right ventricular hypertrophy, atrial enlargement, aortic root dilatation, valvar thickening (both aortic and mitral valves), mitral valve prolapse, and restrictive and hypertrophic cardiomyopathy. ${ }^{6-10}$ The increased ventricular wall thickness is the consequence of the deposition of globotriaosylceramide in cardiomyocytes and cellular hypertrophy. In addition, coronary artery disease was reported. ${ }^{11}$ In general, hemizygous males suffer at a young age from more abnormalities than heterozygous females. Furthermore, hypertrophy and conduction disorders are usually more advanced with a longer duration of the disease process.

\section{FABRY DISEASE AS A CAUSE OF HYPERTROPHIC CARDIOMYOPATHY}

In two recent observational studies, ${ }^{89}$ Fabry disease was diagnosed in $3-4 \%$ of older men (mean (SD) age 65 (6) and 53 (8) years, respectively) with LV hypertrophy. In both studies, patients with the disease had reduced plasma $\alpha$ galactosidase A activity (normal $>1.2 \mathrm{nmol} / \mathrm{h} /$ $\mathrm{ml}$ ). In this issue of Heart, Ommen and colleagues report the histopathological findings in 100 consecutive patients (51 (16) years) with hypertrophic obstructive cardiomyopathy who underwent surgical myectomy. ${ }^{12}$ Unlike previous studies, there were no patients with Fabry disease as inferred from the pathological examination of the resected septal tissue. There are a number of potential reasons for the apparently discordant findings with previous reports. First, the patients in Ommen and colleague's study had a younger age than those included by Nakao and colleagues, ${ }^{8}$ and it is generally accepted that the likelihood and severity of cardiac involvement increase with age, possibly accounting for the lack of cases in the current series. However, Sachdev and colleagues" included patients with a very similar mean age to those in the current series. Furthermore, considering the standard deviation of all three studies, ${ }^{8}{ }^{12}$ there is a large overlap in the patients' age. Therefore, age is not the main reason for the different findings.

Second, the disease is most severe in hemizygous males and there were 66 femaleswho should have a lower likelihood and severity of the disease-in the current study. While this line of reasoning has theoretical and clinical support, a recent study ${ }^{10}$ of 55 heterozygous females (mean age 40 years) found LV hypertrophy in 35 patients (64\%). Third, in the three largest studies, ${ }^{8-10}$ LV outflow tract obstruction was present in only three of 69 patients $(4 \%)$. The current surgical series included only patients with severe symptoms and outflow tract obstruction who were undergoing myectomy, thus potentially 
excluding patients with Fabry disease who appear to have a low incidence of outflow tract obstruction. Fourth, judging by the larger studies, ${ }^{8-10}$ concentric LV hypertrophy is the most common pattern of hypertrophy in patients with Fabry disease, whereas the patients in the report by Ommen and colleagues $^{12}$ exhibited asymmetric LV hypertrophy. Finally, if the histopathological findings of Fabry disease occur in a patchy pattern, it is conceivable that the examination of only the septal myocardium could result in missing some cases. In summary, considering the above four largest studies with 538 patients, it is reasonable to recommend screening for Fabry disease in individuals with unexplained concentric LV hypertrophy, particularly in those without severe LV outflow tract obstruction.

\section{IMPLICATIONS OF A POSITIVE DIAGNOSIS OF FABRY DISEASE}

In addition to genetic counselling, there are important treatment considerations. In patients with classic disease, enzyme replacement therapy was recently shown in randomised controlled trials to result in the clearance of microvascular endothelial deposits of globotriaosylceramide. ${ }^{13}$ Likewise, the use of intravenous galactose in a patient with isolated cardiac involvement resulted in a dramatic improvement of cardiac function. Before treatment, the patient was in New York Heart Association functional class IV and was under consideration for cardiac transplantation. After therapy, LV ejection fraction increased from $31 \%$ to $55 \%$ over a two year period along with a dramatic improvement in symptomatology. ${ }^{3}$ In addition, gene therapy appears to have a great potential for treating patients afflicted with this disease. In mice reproducing the multiorgan involvement of Fabry disease, the delivery of recombinant adeno-associated viral vector encoding human $\alpha$ galactosidase A into the hepatic portal vein resulted in an improvement of enzymatic and functional abnormalities. ${ }^{14}$ It is therefore incumbent upon physicians evaluating cardiac patients with unexplained LV hypertrophy to consider Fabry disease in the differential diagnosis.

While the above studies have advanced our understanding with respect to the cardiac diagnosis and management of this disease, a number of important questions remain. Is it possible to identify patients with cardiac disease at a stage preceding LV hypertrophy? This is particularly important, since some patients can have lysosomal storage of globotriaosylceramide in the absence of hypertrophy by ECG and/or echocardiography. Furthermore, it is possible that intervening with enzyme therapy at an earlier-perhaps preclinical-stage could result in a more effective and potentially curative treatment. The diagnostic criterion of cardiac involvement in heterozygous females is another area in need of study. The enzyme values of these patients overlap with those of normal individuals, reducing the clinical utility of this approach. Likewise, because many of the heterozygous females will not manifest clinical disease until later in life, conventional echocardiography may have a somewhat limited role as it can identify only patients with more advanced disease. In addition, future studies should examine the incidence of Fabry disease in a community based population (and not tertiary referral centres) of hypertrophic cardiomyopathy.

A recent report by Pieroni and colleagues ${ }^{15}$ has shown that it is possible to detect subjects with positive mutations for Fabry disease but without LV hypertrophy by tissue Doppler imaging of the mitral annulus. The investigators noted that septal and lateral systolic and early diastolic velocities at both sides of the mitral annulus were highly accurate in identifying mutation positive subjects without LV hypertrophy. This study sets the stage for potential clinical trials considering such subjects for enzyme replacement therapy.

\section{ACKNOWLEDGEMENTS}

Supported by an American Heart Association Scientist Development grant $(0030235 \mathrm{~N})$ from the AHA National Center, Dallas Texas.

\section{REFERENCES}

1 Desnick RJ, loannou YA, Eng CM. Alpha galactosidase A deficiency: Fabry disease. In: Scriver CR, Beaudet AL, Sly WS, Valle D, eds. The metabolic and molecular bases of inherited disease. 8th ed, vol 3. New York: McGraw-Hill, 2001:3733-74.

2 Kuznetsov G, Nigam SK. Folding of secretory and membrane proteins. N Engl J Med 1998;339:1688-95.

3 Frustaci A, Chimenti C, Ricci R, et al. Improvement in cardiac function in the cardiac variant of Fabry's disease with galactose-infusion therapy. N Engl J Med 2001;345:25-32.

4 Mehta J, Tuna N, Moller JH, et al. Electrocardiographic and vectorcardiographic abnormalities in Fabry's disease. Am Heart $J$ 1977;93:699-705

5 Doi Y, Toda G, Yano K. Sisters with atypical Fabry's disease with complete atrioventricular block. Heart 2003;89:e2

6 Desnick RJ, Blieden LC, Sharp HL, et al. Cardiac valvular anomalies in Fabry disease: clinical, morphologic, and biochemical studies. Circulation 1976;54:818-25.

7 Bass JL, Shrivastava S, Grabowski GA, et al. The M-mode echocardiogram in Fabry's disease. Am Heart J 1980;100:807-12.

8 Nakao S, Takenaka T, Maeda M, et al. An atypical variant of Fabry's disease in men with left ventricular hypertrophy. N Engl J Med 1995;333:288-93

9 Sachdev B, Takenaka T, Teraguchi H, et al. Prevalence of Anderson-Fabry disease in male patients with late onset hypertrophic cardiomyopathy. Circulation 2002;105:1407-11

10 Kampmann C, Baehner F, Whybra C, et al. Cardiac manifestations of Anderson-Fabry disease in heterozygous females. J Am Coll Cardiol 2002;40: 1668-74.

11 Fisher EA, Desnick R, Gordon RE, et al. Fabry disease: an unusual cause of severe coronary disease in a young man. Ann Intern Med 1992;117:221-3.

12 Ommen SR, Nishimura RA, Edwards WD. Fabry disease: a mimic for obstructive hypertrophic cardiomyopathy? Heart 2003;89:929-30.

13 Eng CM, Guffon N, Wilcox WR, et al. Safety and efficacy of recombinant human alpha galactosidase $A$ replacement in Fabry disease. N Engl J Med 2001;345:9-16.

14 Jung SC, Han IP, Limaye A, et al. Adeno-associated viral vector-mediated gene transfer results in long-term enzymatic and functional correction in multiple organs of Fabry mice. Proc Natl Acad Sci USA 2001;98:2676-81.

15 Pieroni M, Chimenti C, Ricci R, et al. Early detection of Fabry cardiomyopathy by tissue Doppler imaging. Circulation 2003:107: 1978-84. 